\title{
Structure and giant magnetoresistance of granular Co-Cu nanolayers prepared by cross-beam pulsed laser deposition
}

Cite as: J. Appl. Phys. 107, 023904 (2010); https://doi.org/10.1063/1.3277023

Submitted: 20 July 2009. Accepted: 30 November 2009 . Published Online: 20 January 2010

A. Jesche, A. Gorbunoff, A. Mensch, H. Stöcker, A. A. Levin, and D. C. Meyer

ARTICLES YOU MAY BE INTERESTED IN

Giant magnetoresistance in magnetic multilayered nanowires

Applied Physics Letters 65, 2484 (1994); https://doi.org/10.1063/1.112672

Thickness dependence of giant magnetoresistance effect in granular Cu-Co thin films

Journal of Applied Physics 85, 4471 (1999); https://doi.org/10.1063/1.370378

$\mathrm{Ll}_{0}$-ordered FePtAg-C granular thin film for thermally assisted magnetic recording media (invited)

Journal of Applied Physics 109, $07 B 703$ (2011); https://doi.org/10.1063/1.3536794

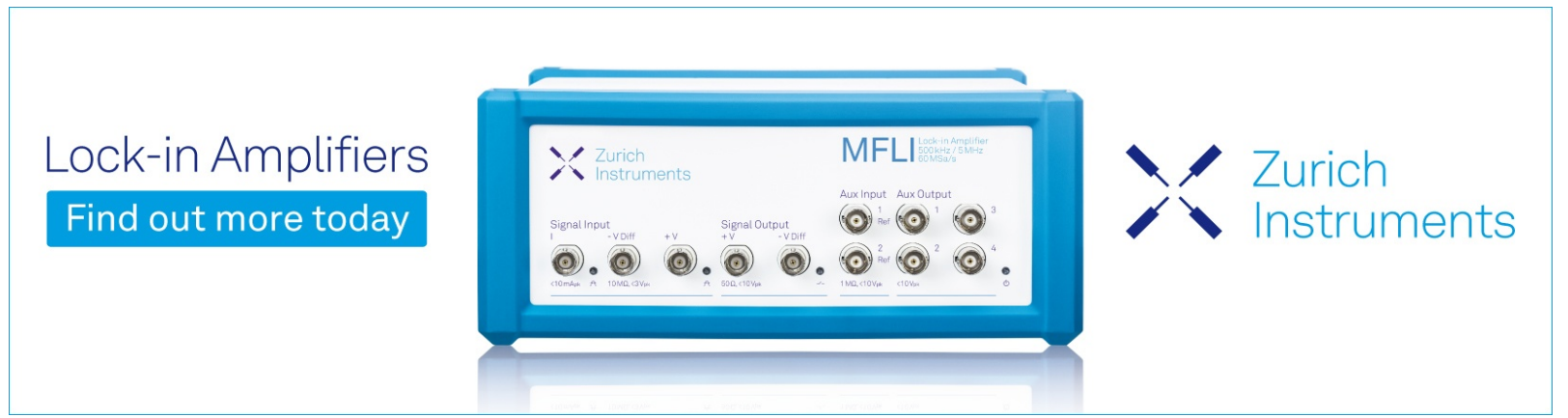




\title{
Structure and giant magnetoresistance of granular $\mathrm{Co}-\mathrm{Cu}$ nanolayers prepared by cross-beam pulsed laser deposition
}

\author{
A. Jesche, ${ }^{1, a)}$ A. Gorbunoff, ${ }^{2}$ A. Mensch, ${ }^{3}$ H. Stöcker, ${ }^{1}$ A. A. Levin, ${ }^{1}$ and D. C. Meyer ${ }^{1}$ \\ ${ }^{1}$ Institut für Strukturphysik, Technische Universität Dresden, D-01062 Dresden, Germany \\ ${ }^{2}$ Hochschule für Technik und Wirtschaft Dresden, D-01069 Dresden, Germany \\ ${ }^{3}$ Institut für Werkstoffwissenschaft, Technische Universität Dresden, D-01062 Dresden, Germany
}

(Received 20 July 2009; accepted 30 November 2009; published online 20 January 2010)

\begin{abstract}
A series of $\mathrm{Co}_{x} \mathrm{Cu}_{100-x}(x=0,40-75,100)$ layers with thicknesses between 13 and $55 \mathrm{~nm}$ were prepared on silicon substrates using cross-beam pulsed laser deposition. Wide-angle $\mathrm{x}$-ray diffraction (WAXRD), transmission electron microscopy (TEM), and electrical transport measurements revealed a structure consisting of decomposed cobalt and copper grains with grain sizes of about $10 \mathrm{~nm}$. The influence of cobalt content and layer thickness on the grain size is discussed. Electron diffraction indicates the presence of an intermetallic $\mathrm{Co}-\mathrm{Cu}$ phase of $\mathrm{Cu}_{3} \mathrm{Au}$ structure type. Thermal treatment at temperatures between 525 and $750 \mathrm{~K}$ results in the progressive decomposition of $\mathrm{Co}$ and $\mathrm{Cu}$, with an increase of the grain sizes up to about $100 \mathrm{~nm}$. This is tunable by controlling the temperature and duration of the anneal, and is directly observable in WAXRD patterns and TEM images. A careful analysis of grain size and the coherence length of the radiation used allows for an accurate interpretation of the x-ray diffraction patterns, by taking into account coherent and noncoherent scattering. The alloy films show a giant magnetoresistance of $1 \%-2.3 \%$ with the maximum obtained after annealing at around 725 K. () 2010 American Institute of Physics. [doi:10.1063/1.3277023]
\end{abstract}

\section{INTRODUCTION}

With the discovery of the giant magnetoresistance (GMR) effect in multilayer ${ }^{1}$ and single-layer ${ }^{2}$ systems, thin films of cobalt and copper have been investigated for basic research and applications. ${ }^{3}$ The value of GMR, which varies between $1 \%$ and $20 \%$ in granular layers of $\mathrm{Co}$ and $\mathrm{Cu}$, depends strongly on the preparation and postprocess annealing. In addition to the electronic properties, these layers are also interesting from the structural point of view. Despite being isomorphic metals with quite similar lattice parameters (cobalt: space group $F m \overline{3} m, a=3.5446 \AA$; copper: $F m \overline{3} m, a$ $=3.6148 \AA)^{4}$ cobalt and copper are immiscible as bulk materials. ${ }^{5}$ However, it is well known that thin layers can behave differently, especially when energetic deposition methods like pulsed laser deposition (PLD) are used. Thus, in addition to the decomposed $\mathrm{Co}$ and $\mathrm{Cu}$ grains ${ }^{6}$ found in thin layers, a solid solution (mixed crystal) ${ }^{7}$ and evidence for an intermetallic alloy ${ }^{8}$ have been reported for $\mathrm{Co}-\mathrm{Cu}$ systems. The use of cross-beam PLD (CB-PLD) for the preparation of alloy $\mathrm{Fe}-\mathrm{Cr}$ nanolayers has led to the discovery of crystalline phases not reported before. ${ }^{9,10}$ Therefore, the aim of the present work was to show whether the formation of metastable phases by using CB-PLD is also observable for $\mathrm{Co}-\mathrm{Cu}$ layers. We present a growth procedure, determine the lattice spacing and grain size, and discuss the effect of a postprocess annealing on structure and magnetoresistance of $\mathrm{Co}-\mathrm{Cu}$ nanolayers with different compositions.

\footnotetext{
${ }^{a)}$ Present address: Max-Planck-Institut für Chemische Physik fester Stoffe, D-01187 Dresden, Germany. Electronic mail: jesche@cpfs.mpg.de.
}

\section{EXPERIMENTAL PROCEDURE}

\section{A. Sample preparation}

The $\mathrm{Co}-\mathrm{Cu}$ alloy films were prepared by CB-PLD. A detailed description of this technique can be found in Ref. 11. CB-PLD allows the simultaneous and droplet-free deposition of two materials, in our case, $\mathrm{Co}$ and $\mathrm{Cu}$. In this technique, after ablation from two separated targets, the materials are mixed to variable proportions in the colliding laser plumes, thereby resulting in a mixed $\mathrm{Co}-\mathrm{Cu}$ plume that is directed toward the substrate. Thermally oxidized (001) silicon wafers with a thickness of the amorphous silicon oxide layer of $500 \mathrm{~nm}$ were used as substrates. All depositions were performed at room temperature (RT) in a high vacuum (pressure $p<10^{-6}$ mbar). A neodymium doped yttrium aluminum garnet laser with a wavelength of $\lambda=1064 \mathrm{~nm}$ and an intensity of $\approx 1 \mathrm{GW} / \mathrm{cm}^{2}$ at the target was used for the ablation. The repetition rate of the laser was 10 shots/s yielding an average film-deposition rate of $\approx 0.3 \mathrm{~nm} / \mathrm{min}$ on the substrate. In order to allow for uniform ablation, the target was rotated with a speed of $\approx 0.5$ rotations/s combined with a simultaneous periodic translation with a rate of $0.1 \mathrm{~mm} / \mathrm{s}$. The substrate was positioned at a distance of $10 \mathrm{~cm}$ from the target and $3 \mathrm{~cm}$ away from the origin of the resulting laser plume. In order to get a more homogeneous film, the substrate was rotated with a speed of $\approx 1$ rotation/s. By varying the ablation geometry, compositions of $40 \%-75 \%$ cobalt could be realized. A preparation of $\mathrm{Co}-\mathrm{Cu}$ alloy films containing more $\mathrm{Cu}$ is difficult because of the enhanced resputtering of $\mathrm{Cu}$ compared to $\mathrm{Co}$. In addition, pure $\mathrm{Co}$ and $\mathrm{Cu}$ films were prepared.

A postprocess anneal was carried out in a tubular furnace at temperatures from $T=525 \mathrm{~K}$ up to $T=725 \mathrm{~K}$ in steps of 
$25 \mathrm{~K}$ under high-vacuum conditions ( $\left.p \approx 10^{-6} \mathrm{mbar}\right)$. After an anneal duration of $75 \mathrm{~min}$, the samples were cooled down with an average rate of $10 \mathrm{~K} / \mathrm{min}$. A final thermal treatment was carried out at $750 \mathrm{~K}$ for $16 \mathrm{~h}$ in order to reach a stable state.

\section{B. Characterization}

The thickness of the deposited layers was determined by means of x-ray reflectometry to vary between 13 and $55 \mathrm{~nm}$ and did not change significantly after annealing. The lattice spacing and crystallite size of the $\mathrm{Co}-\mathrm{Cu}$ films were determined by wide-angle $x$-ray diffraction (WAXRD) using a D8-Advance diffractometer (Bruker AXS) with $\mathrm{Cu} K \alpha$ radiation in divergent beam geometry. The Si 004 reflection was used as an internal standard for correcting $2 \theta$ angle reflection positions. A coupled (symmetric) $\omega-2 \theta$ scan mode was used, where $\omega$ is the angle of the incident $\mathrm{x}$-ray beam with respect to the surface, $2 \theta$ is the angle between incident and reflected $\mathrm{x}$-ray beam and $\omega=\theta$ for the symmetric scan mode. Therefore, only lattice planes oriented parallel to the sample surface were taken into account in this scan mode. Asymmetric $2 \theta$ scan modes $(\omega$ is fixed at a value corresponding to the maximum intensity of the respective rocking curve measured) did not show any influence on the x-ray reflection positions observed in the corresponding coupled scans. No additional WAXRD reflections were found by asymmetric scans.

The chemical composition of the layers was determined by means of energy dispersive x-ray spectroscopy (EDX) using a semiconductor detector (Noran Voyager 2000). Transmission electron microscopy (TEM) and electrondiffraction (ED) measurements were made in a Zeiss EM 912 OMEGA microscope in order to determine structural parameters, homogeneity and grain size of the films. As transmission of the silicon substrate with the electron beam, or the removal of the deposited layer from the substrate, are impossible, a $\mathrm{NaCl}$ crystal was coated together with the substrate. After deposition of the $\mathrm{Co}-\mathrm{Cu}$ layer, the $\mathrm{NaCl}$ crystal was dissolved in water and the $\mathrm{Co}-\mathrm{Cu}$ layer was put onto a TEM mesh.

The electric transport measurements were performed at temperatures from 10 to $300 \mathrm{~K}$ in magnetic fields up to $\mu_{0} H=12$ T by using a VSM-Maglab cryostat (Oxford Instruments). The resistivity was measured by a standard fourpoint contact method using conductive silver paint. In order to prevent silver contamination, some of the measurements were carried out using a simple two-point contact method. A comparison of both techniques did not show significant differences. The characterization was performed ex situ because phases which are (meta)stable at ambient conditions were the focus of our investigations. Structure and composition did not change at ambient conditions over a period of one year.

\section{RESULTS-AS-PREPARED SAMPLES}

\section{A. Structural parameters obtained by WAXRD}

Typical WAXRD patterns of two characteristic asprepared $\mathrm{Co}-\mathrm{Cu}$ nanolayers with different thickness and composition are shown in Fig. 1. Note, in contrast to the

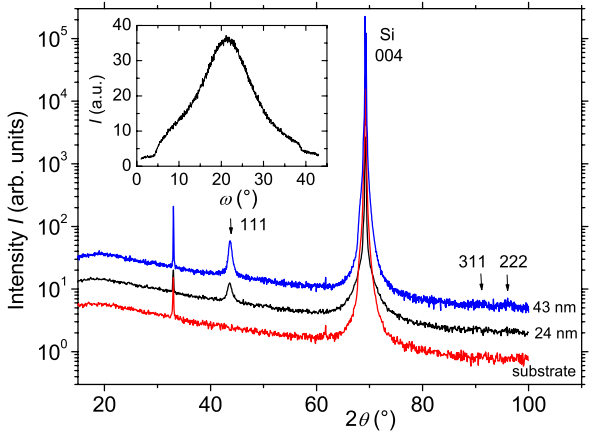

FIG. 1. (Color online) WAXRD patterns of as-prepared $\mathrm{Co}-\mathrm{Cu}$ films with different thickness $t$ and composition $\left(\mathrm{Co}_{59} \mathrm{Cu}_{31}, t=24 \mathrm{~nm}\right.$ and $\mathrm{Co}_{40} \mathrm{Cu}_{60}$, $t=43 \mathrm{~nm})$. For comparison, the WAXRD pattern of the Si substrate is shown (bottom). The reflection indices of the film reflections observed are indicated. The inset shows a typical rocking curve of the 111 reflection of a $\mathrm{Co}_{40} \mathrm{Cu}_{60}$ film.

known $\mathrm{x}$-ray investigations on thin $\mathrm{Co}-\mathrm{Cu}$ films from literature, our layers are rather thin. Due to the preferred orientation (fiber texture) and the small scattering volume of thin films, only a limited number of reflections are observable in the WAXRD patterns. Nevertheless, the WAXRD patterns show reflections not related to the substrate. For all $\mathrm{Co}_{x} \mathrm{Cu}_{100-x}$ compositions investigated, the nonsubstrate reflection with the highest intensity can be indexed as the 111 reflection of the face-centered cubic (fcc) $\mathrm{Co}-\mathrm{Cu}$ lattice. The inset in Fig. 1 shows a rocking curve around this reflection, revealing on the one hand that the texture is strong enough to inhibit the detection of other WAXRD reflections. On the other hand, the rocking curve is broad in comparison to an epitaxially grown layer or a single crystal and, therefore, the intensity of the scattered radiation (for a given $\omega-2 \theta$ coupling) is low. These facts are responsible for the difficulties associated with using XRD for thin layers. Hence, a careful optimization of step width, sampling time, and slits is necessary in order to obtain adequate results.

For layers with a thickness of less than $30 \mathrm{~nm}$, only the 111 reflection of the fcc lattice is observable (see second curve in Fig. 1 as an example). Nevertheless, the crystallographic structure of the layers is assumed to be similar to the structure of bulk fcc $\mathrm{Cu}$ and $\mathrm{Co}$. This approach is supported by the fact that the observed (111) lattice spacing of pure Co and pure $\mathrm{Cu}$ layers is close to the values of the corresponding bulk materials. For the pure Co sample, the observed reflection can correspond either to the 111 reflection of the Co fcc structure or to the 0002 reflection of the Co hexagonal structure. Both structures are known to exist in thin cobalt layers. ${ }^{12-14}$ The upper pattern in Fig. 1 is a typical WAXRD pattern of a layer with a thickness of more than $40 \mathrm{~nm}$ in which weak 311 and $222 \mathrm{Co}-\mathrm{Cu}$ reflections are observed. Their low signal-to-noise ratio does not allow a quantitative analysis of the structural parameters. The lower pattern in Fig. 1 belongs to an uncoated silicon substrate. This shows that the substrate reflections are up to three orders of magnitude higher than those belonging to the $\mathrm{Co}-\mathrm{Cu}$ nanolayers.

Instead of the individual $111 \mathrm{Cu}$ and $111 \mathrm{Co}$ reflections that would be expected due to the immiscibility of the deposited materials, one $111 \mathrm{fcc}$ reflection is clearly observed in the WAXRD patterns of the Co-Cu layers. In general, this 


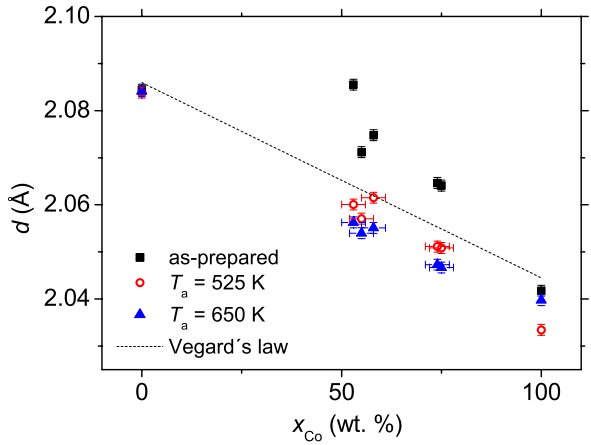

FIG. 2. (Color online) Lattice spacing of the (111) atomic planes of $\mathrm{Co}_{x} \mathrm{Cu}_{100-x}$ layers, as-prepared and after annealing at temperatures indicated (duration of $75 \mathrm{~min}$ ). In the case of the pure Co film, the lattice spacing corresponds either to the (111) atomic plane of the Co fcc structure or to the (0002) atomic plane of the Co hexagonal structure (see text). The ESD of the experimental points are given by the horizontal and vertical bars. For comparison, the lattice spacings calculated according to Vegard's law are shown by the dotted line. For the calculations, the unit-cell parameters of bulk fcc $\mathrm{Cu}$ and $\mathrm{Co}$ were used (Ref. 4).

could be caused by the formation of a metastable $\mathrm{Co}-\mathrm{Cu}$ solid solution or by size effects of the scattering crystallites. The determined (111) lattice spacing obtained for different $\mathrm{Co}_{x} \mathrm{Cu}_{100-x}$ compositions is shown in Fig. 2 together with Vegard's law calculated for a hypothetic $\mathrm{Co}-\mathrm{Cu}$ mixed crystal (see Sec. V). The strong deviation for the $\mathrm{Co}_{51} \mathrm{Cu}_{49}$ composition (as-prepared) from Vegard's law, where the experimental lattice spacing is larger than that of pure copper, renders the existence of a solid solution very unlikely.

It should be noted, that any strain present in the crystallites in the film could contribute to the deviation observed. By assuming the absence of strain in films after annealing at temperatures higher than $525 \mathrm{~K}$ (see Fig. 2), the strain in as-prepared films was estimated from the lattice parameter change to be $0.5 \%-1.5 \%$. An attempt to estimate the crystallite size in as-prepared films, based on these strain values, led to an unrealistic model of infinite crystallites (i.e., the strain was overestimated). Thus, the strain is significantly smaller than $0.5 \%$ and could not be evaluated accurately using the data obtained.

If strain is neglected, the crystallite size $D$ for $\mathrm{Co}_{x} \mathrm{Cu}_{100-x}$ films can be estimated by using the Scherrer equation

$$
D=0.9 \lambda /(B \cos \theta)
$$

where $B$ is the full width at half maximum (FWHM) of the WAXRD reflection. In order to take into account the instrumental broadening caused by the experimental setup, the observed FWHM $\left(\mathrm{FWHM}_{\mathrm{obs}}\right)$ of the WAXRD reflection was corrected by using the FWHM of the silicon 004 substrate reflection $\left(\mathrm{FWHM}_{\text {instr }}\right)$. Since for all reflections $\mathrm{FWHM}_{\mathrm{obs}} / \beta<0.64$ ( $\beta$ is the integral breadth of the reflection), the reflection profiles could be described as Lorentzians. ${ }^{15}$ Following the procedure described in $^{16}$ for $\mathrm{x}$-ray reflections of Lorentzian profile shape, the reflection FWHM was corrected by

$$
B=\mathrm{FWHM}_{\mathrm{obs}}-\mathrm{FWHM}_{\mathrm{instr}} \text {. }
$$
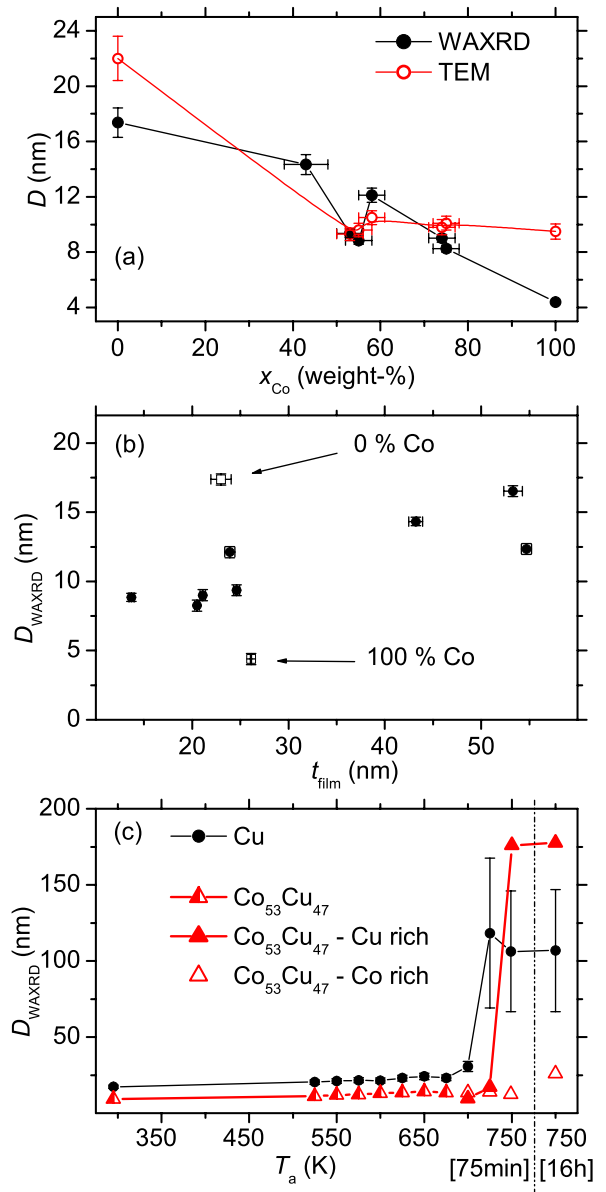

FIG. 3. (Color online) (a) Comparison of the crystallite sizes $D$ calculated by the Scherrer formula from WAXRD data and grain sizes measured by TEM for as-prepared $\mathrm{Co}_{x} \mathrm{Cu}_{100-x}$ films. (b) Crystallite size in as-prepared films as a function of the layer thickness. (c) Development of the crystallite size after annealing at temperatures $T_{a}$ for $\mathrm{Co}_{53} \mathrm{Cu}_{47}$ and pure $\mathrm{Cu}$ layers (see Sec. IV A). The lines in (a) and (c) are guides to the eye. The ESDs of the experimental points are shown by horizontal and vertical bars.

The crystallite sizes $\left(D_{\text {WAXRD }}\right.$ ) obtained for different compositions, together with the grain size obtained by TEM $\left(D_{\text {TEM }}\right.$, see next paragraph), are shown in Fig. 3(a). The crystallite/grain size does not exceed the thickness of the layers. We measured the thickness of the sample containing $53 \%$ Co to be $25 \mathrm{~nm}$, whereas the thickness of the sample containing 55\% Co is only $13 \mathrm{~nm}$. Nevertheless, their crystallite sizes are very similar [Fig. 3(a)], which shows that the crystallite size is more strongly affected by the Co content than by the layer thickness. However, there is a weak correlation between layer thickness and crystallite size observable [Fig. 3(b)]. Within the accuracy of our measurements the crystallite/grain sizes of the alloy systems lie between the grain sizes of the pure compounds.

\section{B. TEM}

Another approach used to measure the crystal grain sizes was TEM. The dark grains in the TEM micrographs (Fig. 4) fulfill the Bragg condition, i.e., the contrast is not induced by absorption differences. The grain size was determined by averaging the size of 20 randomly chosen grains. These are shown in Fig. 3(a) as a function of cobalt content. It should 


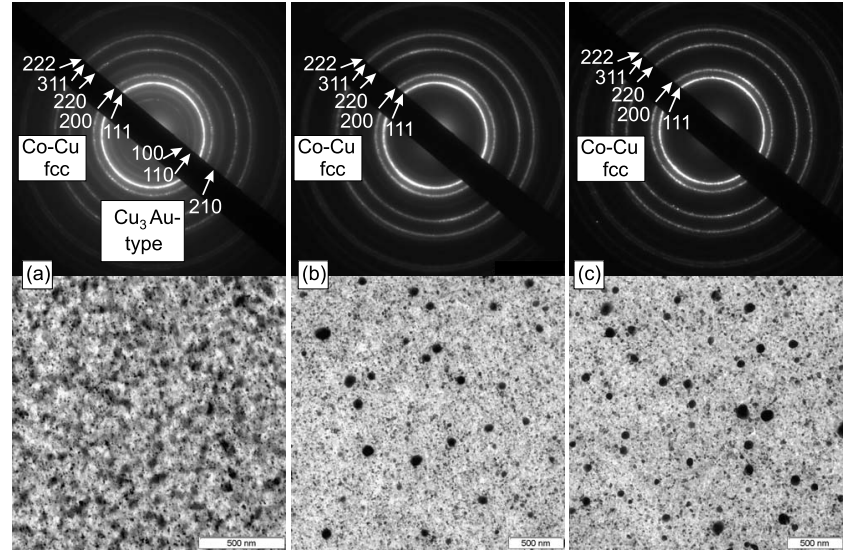

FIG. 4. TEM bright field images (lower part) and ED patterns (upper part) of a $\mathrm{Co}_{59} \mathrm{Cu}_{41}$ layer (a) as-prepared, (b) after thermal treatment for $15 \mathrm{~min}$ at $725 \mathrm{~K}$, and (c) after thermal treatment at $875 \mathrm{~K}$. The diffraction rings not belonging to the fcc lattice disappear after thermal treatment at $725 \mathrm{~K}$.

be noted that the grain size determined by TEM is in most cases not the same quantity as the crystallite size obtained by WAXRD. The grains can contain more than one crystallite. As can be seen in Fig. 3(a), for all alloy compositions, the size of crystallites and grains determined by WAXRD and TEM, respectively, are equal in the limits of the estimated standard deviations (ESDs). This highlights the equivalence of the crystallite and grain sizes for the deposited alloy $\mathrm{Co}_{x} \mathrm{Cu}_{100-x}$ nanolayers. At the same time, the grain sizes of the pure $\mathrm{Co}$ and $\mathrm{Cu}$ films are about two to three times larger than the crystallite sizes, indicating the formation of large grains containing two to three crystallites.

ED patterns [Fig. 4(a)] show the Bragg reflections which can be well indexed using a fcc lattice in agreement with the WAXRD patterns. Three additional reflections violating the extinction law of the fcc lattice were found for all compositions (as-prepared films). The assumption of an additional intermetallic phase of $\mathrm{Cu}_{3} \mathrm{Au}$-type and unit-cell parameter $a=3.55 \AA$, theoretically predicted by Yan et al. ${ }^{8}$ is in good agreement with the experimental data. As the $\mathrm{Cu}_{3} \mathrm{Au}$ structure-type is a superstructure of the fcc lattice, the similar atomic scattering factors of $\mathrm{Co}$ and $\mathrm{Cu}$ cause a weak intensity of the 100,110, and 210 Bragg reflections which cannot be resolved by WAXRD. However, the different substrate $(\mathrm{NaCl})$ also could be responsible for the occurrence of these phases compared to the case of the WAXRD investigated layers on amorphous silicon oxide substrates.

\section{Electrical transport measurements-GMR}

The electrical transport measurements are shown in Fig. 5 for nanolayered $\mathrm{Co}_{59} \mathrm{Cu}_{41}$ (as-prepared). The development of the electrical resistance at RT as a function of applied magnetic field is shown in Fig. 5(a) for two different orientations of the field. The change in the resistivity $\Delta \rho$ is defined as

$$
\Delta \rho=\frac{\rho(B)-\rho(B=0)}{\rho(B=0)} .
$$

For a magnetic field oriented perpendicular to the surface and thus perpendicular to the electrical current, the resistivity
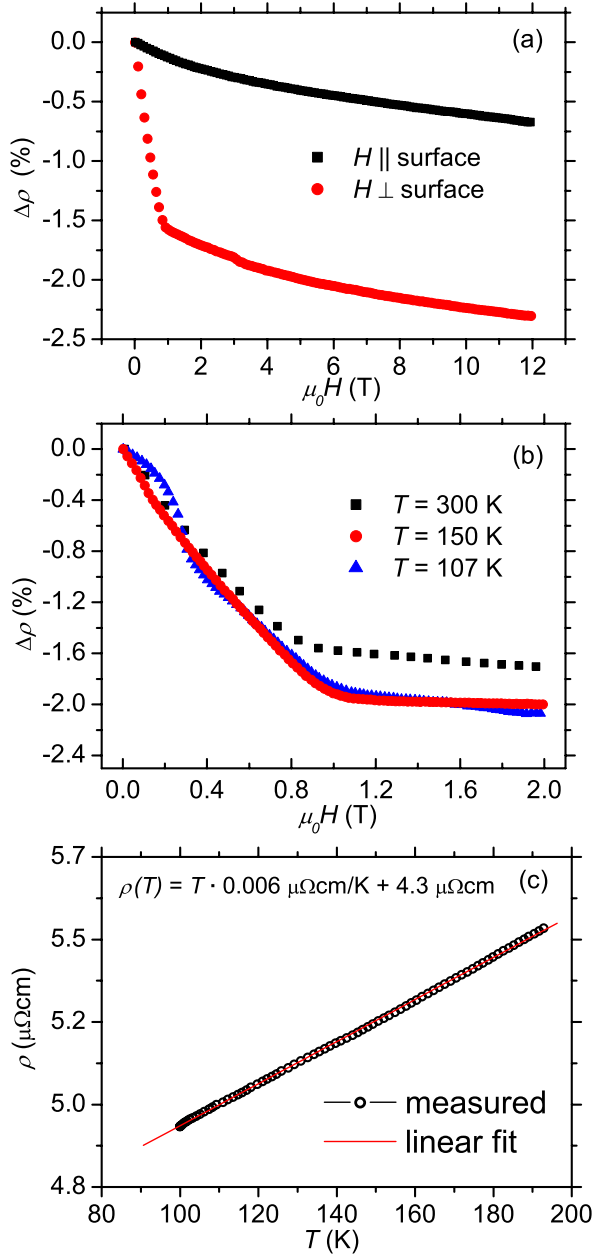

FIG. 5. (Color online) Nanolayer $\mathrm{Co}_{59} \mathrm{Cu}_{41}$. (a) Change of the electrical resistivity $\Delta \rho$ at RT for magnetic fields perpendicular and parallel to the surface. (b) Change in the electrical resistivity as a function of applied magnetic field for three different temperatures. The magnetic field was oriented perpendicular to the sample surface. (c) The electrical resistivity as a function of temperature in zero magnetic field.

rapidly decreases by $\Delta \rho=-1.6 \%$ at $\mu_{0} H=0.9 \mathrm{~T}$ followed by a moderate decrease to $\Delta \rho=-2.3 \%$ at $\mu_{0} H=12$ T. This decrease is most likely caused by the GMR effect, because the anisotropic magnetoresistance exhibits a smaller decrease in the resistivity and smaller saturation fields. Literature values for a pure Co layer prepared by PLD are $\Delta \rho=0.5 \%$, with a saturation field of $\mu_{0} H=0.03 \mathrm{~T} .{ }^{17}$ The stronger GMR for a magnetic field aligned perpendicular to the surface is a thinfilm phenomenon and/or caused by the texture (the granular GMR should be isotropic). Even for high fields $\mu_{0} H$ $\approx 12 \mathrm{~T}$ no saturation is observed, which is typical for the GMR in granular layers.

For the following measurements, the magnetic field was applied perpendicular to the surface. Magnetic field dependencies of the GMR effect at RT and lower temperatures are shown in Fig. 5(b). Up to an applied magnetic field of $\mu_{0} H$ $=0.8 \mathrm{~T}$, at RT and low temperature the resistivity change shows approximately the same magnitude. However, the low temperature $(150$ and $107 \mathrm{~K})$ resistivity continues to decrease with the same rate up to a magnetic field of $\mu_{0} H$ $=1 \mathrm{~T}$ reaching $\Delta \rho=-1.95 \%$ with a further moderate decrease to $\Delta \rho=-2.0 \%$ at $\mu_{0} H=2 \mathrm{~T}$. At a temperature of $T$ 


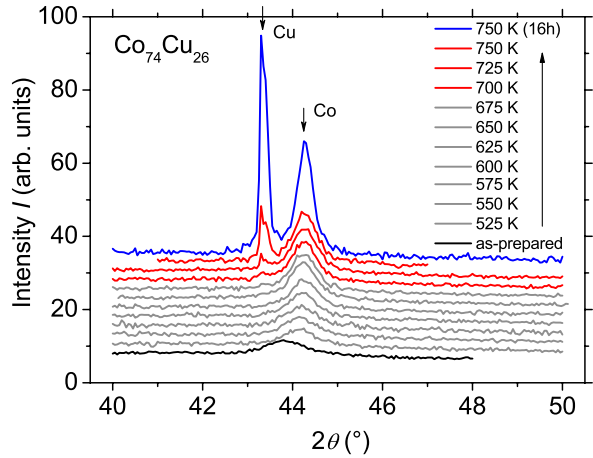

FIG. 6. (Color online) Development of the diffraction pattern ( $2 \theta$ range near the $111 \mathrm{Co}-\mathrm{Cu}$ reflection) for a $\mathrm{Co}_{74} \mathrm{Cu}_{26}$ film after annealing at the temperatures indicated. The annealing time was $75 \mathrm{~min}$ except for the topmost pattern $(16 \mathrm{~h})$. All the diffraction patterns were taken at RT.

$=107 \mathrm{~K}, \Delta \rho$ shows oscillations around the smoothly decreasing GMR curve measured at $T=150 \mathrm{~K}$. However, the temperature was no less stable in this case. We have no explanation for this effect.

The temperature dependence of the electrical resistance in zero magnetic field clearly shows a metal-like behavior [Fig. 5(c)] for temperatures between 100 and $200 \mathrm{~K}$ with an extrapolated residual resistivity of $4.3 \mu \Omega \mathrm{cm}$.

\section{RESULTS-INFLUENCE OF THERMAL TREATMENT}

\section{A. Development of structural parameters observed by XRD}

The development of the XRD pattern due to thermal treatment is shown in Fig. 6. The corresponding lattice spacings are shown in Fig. 7, where the monotonic decrease of the lattice spacing $d_{111}$ of the $\mathrm{Co}_{x} \mathrm{Cu}_{100-x}$ alloy nanolayers with increasing annealing temperature for $T_{a}<675 \mathrm{~K}$ and for Co-rich grains to higher temperatures is apparent (see solid symbols for alloy compositions in Fig. 7). As a result of annealing process, the interplanar distances $d_{111}$ of the

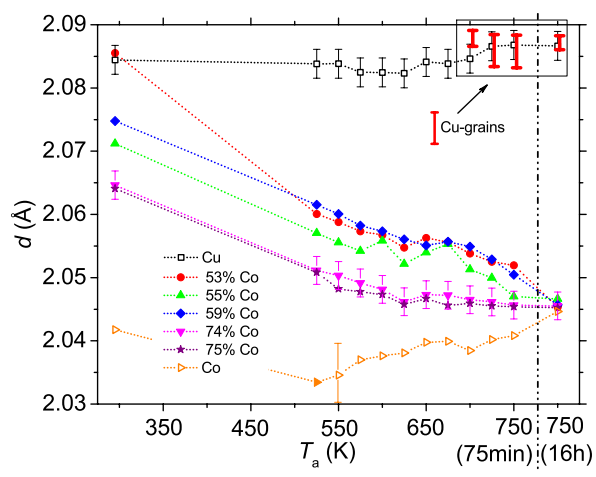

FIG. 7. (Color online) Development of the (111) lattice spacing of $\mathrm{Co}_{x} \mathrm{Cu}_{100-x}$ nanolayers for different Co concentrations as a function of annealing temperature $T_{a}$. The observed lattice spacings cannot be attributed to a solid solution (see discussion). For $T_{a}>675 \mathrm{~K}$ the used values are obtained from the right reflection $(111 \mathrm{Co})$ in the WAXRD pattern (see Fig. 6) and are represented by solid symbols similar to $T_{a}<675 \mathrm{~K}$. For $T_{a}$ $>675 \mathrm{~K}$ the values determined from the left reflection $(111 \mathrm{Cu})$ in the WAXRD pattern (see Fig. 6) do not change significantly and are indicated by thick (red) bars (upper right part, framed). The lines are guides to the eye. ESDs of the experimental points are shown by the vertical bars for pure $\mathrm{Cu}$, $\mathrm{Co}$, and $\mathrm{Co}_{74} \mathrm{Cu}_{26}$.
$\mathrm{Co}_{x} \mathrm{Cu}_{100-x}$ alloy nanolayers show a better agreement with Vegard's law, with values slightly smaller than could be expected from the law (see Fig. 2 as an example for annealing at $T_{a}=525$ and $T_{a}=650 \mathrm{~K}$ ). These results show that it is also possible to adjust the (average) (111) lattice spacing of the material system $\mathrm{Co}-\mathrm{Cu}$ with subpicometer precision by using a thermal treatment.

The development of the lattice spacing belonging to copper-rich grains is shown in the upper right part of Fig. 7, where all measured values are within the thick (red) bars. The corresponding WAXRD reflection, appearing after a thermal treatment at $700 \mathrm{~K}$ (Fig. 6), does not undergo a significant change in $2 \theta$ for the last four thermal treatments but its intensity does. The lattice spacing calculated for this reflection corresponds to pure copper, where the presence of copper has been verified by selected area EDX. The two resulting reflections formed after the final thermal treatment belong to pure cobalt and pure copper. Figure 3(c) shows the development of the crystallite size as a function of the annealing temperature for a pure copper sample and for $\mathrm{Co}_{53} \mathrm{Cu}_{47}$. Up to $T_{a}=675 \mathrm{~K}$ the crystallite size shows a moderate increase with increasing temperature. This is followed by a significant growth of Cu-rich crystallites after higher temperature anneals, where the individual reflections of Coand $\mathrm{Cu}$-rich grains are formed (see Fig. 6).

Note that after annealing at temperatures higher than 700 $\mathrm{K}$, the crystallite/grain sizes determined by WAXRD and TEM exceed the film thickness. However, this possible contradiction is resolved taking into account that only lateral grain size is accessible by TEM. In the case of WAXRD, the calculated crystallite size is a mean effective quantity averaging the crystallite sizes in different directions.

\section{B. Thermal treatment during TEM investigations}

An additional thermal treatment test was performed in the TEM for temperatures between 450 and $600 \mathrm{~K}$. After 15 min annealing, a bright field image and an ED pattern were taken after the sample had cooled down to RT. An increase in the grain size during the thermal treatment is observable from the lower part of Fig. 4. The dark regions, with a size of about $100 \mathrm{~nm}$, belong to grains of pure $\mathrm{Cu}$, as verified by selected area EDX. Similar values for the crystallite sizes of $\mathrm{Cu}$-rich grains after annealing are obtained by XRD using the Scherrer equation [Fig. 3(c)]. In the upper part of Fig. 4 the corresponding ED patterns are shown. It can be seen that the additional rings, which do not belong to the fcc lattice, and are attributed to an intermetallic phase of $\mathrm{Cu}_{3} \mathrm{Au}$ structure-type (see Sec. III B), disappear completely during the thermal treatment.

\section{Influence of thermal treatment on the GMR}

No direct correlation between the composition of the asprepared or annealed layers and the magnitude of the GMR effect was found for the samples investigated in this paper. The change of the GMR effect after a thermal treatment is shown in Fig. 8(a) for a nanolayer with composition $\mathrm{Co}_{75} \mathrm{Cu}_{25}$. The effect increases for anneals of up to $750 \mathrm{~K}$ for $75 \mathrm{~min}$. Further annealing at $750 \mathrm{~K}$ for $16 \mathrm{~h}$ leads to a de- 

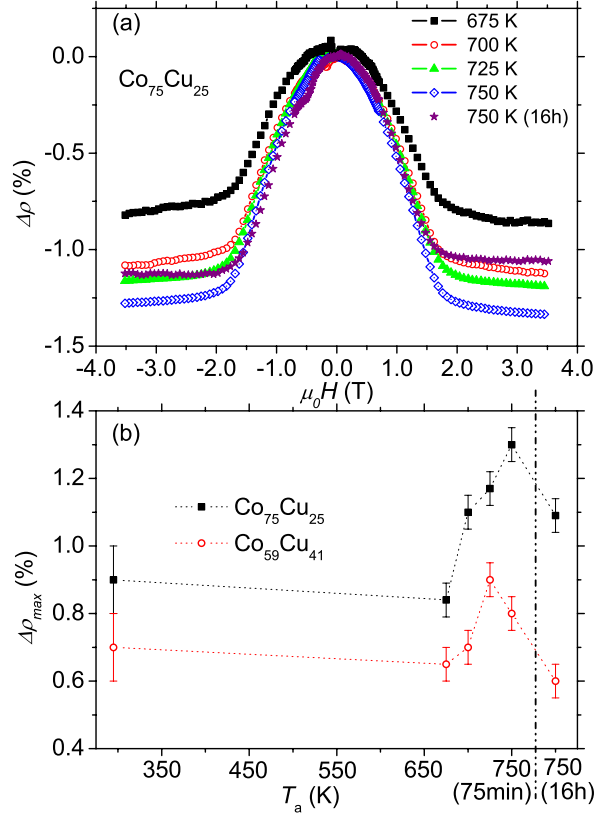

FIG. 8. (Color online) (a) Dependence of $\Delta \rho$ on $\mu_{0} H$ after annealing at given temperatures $T_{a}$. (b) Maximum $\Delta \rho_{\max }=\Delta \rho\left(\mu_{0} H=3.5 \mathrm{~T}\right)$ as a function of annealing temperature $T_{a}$. The duration of thermal treatment was $75 \mathrm{~min}$ per step except for the $16 \mathrm{~h}$ for the last anneal. The lines in (b) are guides for the eye only. ESDs of the experimental points are shown in (b) by the vertical bars. The resistivity measurements in (a) and (b) were performed at RT.

crease in the effect. Figure 8(b) shows the maximum change of the electrical resistivity as a function of the annealing temperature for two samples with characteristic compositions of $59 \% \mathrm{Co}$ and $75 \% \mathrm{Co}$, and a film thickness of about $20 \mathrm{~nm}$. Both samples show a maximum in the GMR effect after annealing at $\approx 725 \mathrm{~K}$. The magnitude of the GMR effect after a thermal treatment is a factor of $\approx 1.5$ larger than the as-prepared samples. This change is rather weak compared to other results published which show comparable values for the as-prepared samples. ${ }^{18,19}$ However, the layer thickness was $\approx 100$ and $200 \mathrm{~nm},{ }^{18,19}$ and annealing led to an enhancement of the GMR by a factor of $\approx 8$ and $\approx 4$, respectively. This suggests the grain size perpendicular to the electrical current plays an important role. A second possible reason for the comparatively small GMR in our case is the large Co content well above the percolation threshold.

\section{DISCUSSION}

All investigated as-prepared $\mathrm{Co}-\mathrm{Cu}$ layers exhibit only one significant Bragg reflection and possess a GMR effect at the same time. Similar observations were published several times, see e.g. ${ }^{18}$ These two points are contradictory because ferromagnetic grains separated by a nonmagnetic spacer, i.e., two separate phases, are necessary for the occurrence of the GMR effect. However, for this case, at least two Bragg reflections of two different crystalline phases, namely one for $\mathrm{Co}$ and one for $\mathrm{Cu}$, would be expected in the WAXRD pattern, which was not the case. Furthermore, the development of the diffraction pattern after the thermal treatments (Fig. 6) could be misinterpreted as a decomposition of an initial solid solution into grains of pure $\mathrm{Co}$ and pure $\mathrm{Cu}$.

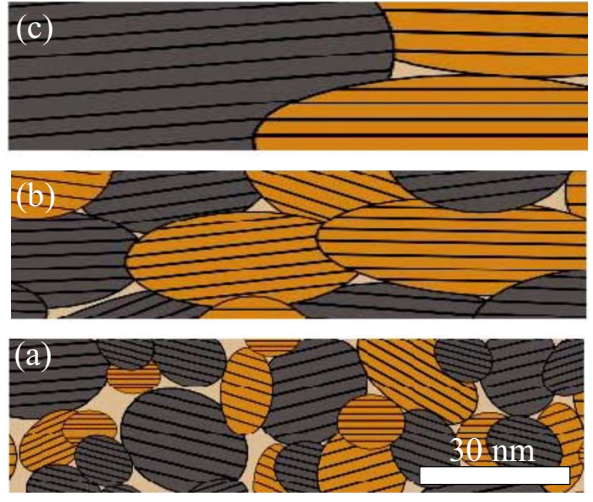

FIG. 9. (Color online) Schematic plot of real structure and its development due to a thermal treatment for a granular $\mathrm{Co}-\mathrm{Cu}$ nanolayer ( $\mathrm{Co}$ and $\mathrm{Cu}$ grains are shown by dark gray and light orange colors, respectively). (a) as-prepared film with fine grains of $\mathrm{Co}$ and $\mathrm{Cu}$ with mean size $D$ less than the coherence length $l$ of the x-ray radiation, (b) film after annealing at $T$ $=700 \mathrm{~K}$ with $\mathrm{Co}$ and $\mathrm{Cu}$ grain mean size $D$ of about $l$, and (c) film after annealing at $T=750 \mathrm{~K}$ with $\mathrm{Co}$ and $\mathrm{Cu}$ grain mean size $D$ larger than $l$.

These discrepancies can be solved by taking into account that the grain size of $\approx 10 \mathrm{~nm}$ is smaller than the coherence length $l$ of the used x-ray radiation. The lateral coherence length $l$ of the $\mathrm{x}$-ray radiation can be estimated using the equation ${ }^{20} l=s \cdot \lambda /(2 \pi B)$, where $\lambda=1.54056 \AA$ is the wavelength used, $s=0.22 \mathrm{~m}$ is the distance between the $\mathrm{x}$-ray source and sample, and $B=0.04 \mathrm{~mm}$ is the $\mathrm{x}$-ray focus spot size (Bruker KFL-Cu-2K tube). From the equation $l$ $\approx 135 \mathrm{~nm}$ is calculated. Therefore, a coherent superposition of $\mathrm{x}$-ray waves scattered by $\mathrm{Co}$ grains and $\mathrm{Cu}$ grains, results in only one Bragg reflection in the diffraction pattern (i.e., the scattered amplitudes of $\mathrm{Co}$ and $\mathrm{Cu}$ are added but not their squares ${ }^{21}$ ). This leads to the misinterpretation of the existence of a solid solution (mixed crystal) in the $\mathrm{Co}-\mathrm{Cu}$ layer. During the thermal treatment, the increase of grain size leads to the development of two Bragg reflections of Co- and $\mathrm{Cu}-$ rich phases in the WAXRD pattern as soon as the grain size exceeds the coherence length of the radiation. This is supported by TEM results which show $\mathrm{Cu}$ grains larger than 100 nm [lower panel in Fig. 4(b)]. Furthermore, the size of the $\mathrm{Cu}$ crystallites is estimated to be larger than $100 \mathrm{~nm}$ using the Scherrer equation [Fig. 3(c)] with the FWHM of the developing 2nd Bragg reflection attributed to pure $\mathrm{Cu}$ (Fig. 6, $T_{a}>700 \mathrm{~K}$, left WAXRD reflection). Therefore, the change of lattice spacings shown in Fig. 7 is not an intrinsic property of the Co-Cu layers but is caused by a complex change of the interference conditions due to increasing grain size. As a matter of fact, the lattice spacing of $\mathrm{Co}$ and $\mathrm{Cu}$ grains in the alloy films does not change significantly during the annealing process, similar to the lattice spacing of single phase Co and $\mathrm{Cu}$ films.

A model of the real structure of $\mathrm{Co}-\mathrm{Cu}$ nanolayers and their development after thermal treatment is schematically represented in Fig. 9. The as-prepared film material consists of fine $\mathrm{Co}$ and $\mathrm{Cu}$ grains, which are smaller than the coherence length of the $\mathrm{x}$-ray radiation as supported by TEM and WAXRD measurements. As a result, the WAXRD patterns exhibit only one Bragg reflection, the electrical resistivity measurements reveal the presence of the GMR effect. Ther- 
mal treatment leads to an increase of the grain size and enhanced GMR values. When the (lateral) grain size reaches the coherence length of the X-ray radiation [Fig. 9(b)], a second Bragg reflection is observed which corresponds to the lattice spacing of pure $\mathrm{Cu}$. Annealing at higher temperatures results in a further increase of (lateral) grain size [Fig. 9(c)] and an increase in GMR effect. However, prolonged annealing at high temperatures results in a decrease of GMR [Fig. 8(b)], which could be caused by an enhanced spin-flip scattering inside larger crystallites. ${ }^{22}$ Finally, for higher annealing temperatures, two distinct Bragg reflections occur in WAXRD patterns and large $\mathrm{Cu}$ grains become visible in the TEM bright field images.

\section{CONCLUSIONS}

The as-prepared film material consists of fine $\mathrm{Co}$ and $\mathrm{Cu}$ grains with typical sizes smaller than the coherence length of the $\mathrm{x}$-ray radiation $(\mathrm{Cu} K \alpha)$ used. This gives an explanation for the occurrence of the GMR effect in as-prepared and annealed films, even though x-ray diffraction patterns show only one Bragg reflection. The size of crystallites in films is more strongly affected by the Co content than by the layer thickness. No intermetallic alloy was found in as-prepared and annealed $\mathrm{Co}-\mathrm{Cu}$ films on silicon substrates using WAXRD. However, indications for an intermetallic alloy of $\mathrm{Cu}_{3} \mathrm{Au}$ structure-type in as-prepared $\mathrm{Co}-\mathrm{Cu}$ films were observed in ED patterns, showing that this phase exists, as theoretically predicted in Ref. 8. Annealing of the samples results in the growth of larger $\mathrm{Co}$ - and $\mathrm{Cu}$-rich grains and a larger GMR effect. However, the appearance of larger GMR values is assumed to be restricted by the small grain size perpendicular to the electrical current, i.e., by the layer thickness.

\section{ACKNOWLEDGMENTS}

The authors would like to thank M. Dörr and S. Granovsky for help with electrical transport measurements and $\mathrm{R}$. Boucher for valuable discussions.

${ }^{1}$ P. Grünberg, R. Schreiber, Y. Pang, M. B. Brodsky, and H. Sowers, Phys. Rev. Lett. 57, 2442 (1986)

${ }^{2}$ J. Q. Xiao, J. S. Jiang, and C. L. Chien, Phys. Rev. Lett. 68, 3749 (1992).

${ }^{3}$ Y. Chen, Y. Mei, R. Kaltofen, J. I. Mönch, J. Schumann, J. Freudenberger, H. J. Klauß, and O. G. Schmidt, Adv. Mater. 20, 3224 (2008).

${ }^{4}$ P. Villars and L. D. Calvert, Pearson's Handbook of Crystallographic Data for Intermetallic Phases (ASM International, Ohio, 1985), Vol. 2.

${ }^{5}$ T. B. Massalski, Binary Alloy Phase Diagrams (ASM International, Ohio, 1986).

${ }^{6}$ A. G. Prieto, M. L. Fdez-Gubieda, C. Meneghini, and A. Garcia-Arribas, J. Appl. Phys. 91, 8596 (2002).

${ }^{7}$ A. N. Pohorilyi, A. F. Kravetz, E. V. Shipil, A. Y. Vovk, C. S. Kim, and H. R. Khan, J. Magn. Magn. Mater. 186, 87 (1998).

${ }^{8}$ H. F. Yan, Y. X. Shen, H. B. Guo, and B. X. Liu, J. Phys.: Condens. Matter 19, 026219 (2007)

${ }^{9}$ A. Gorbunov, A. A. Levin, A. Mensch, D. C. Meyer, A. Tselev, P. Paufler, W. Pompe, and D. Eckert, Appl. Surf. Sci. 197-198, 475 (2002).

${ }^{10}$ A. A. Levin, D. C. Meyer, A. Gorbunov, A. Tselev, W. Pompe, and P. Paufler, J. Alloys Compd. 347, 171 (2002).

${ }^{11}$ A. Gorbunoff, Laser-Assisted Synthesis of Nanostructured Materials (VDI Verlag, Düsseldorf, 2002).

${ }^{12}$ F. J. Lamelas, C. H. Lee, H. He, W. Vavra, and R. Clarke, Phys. Rev. B 40, 5837 (1989)

${ }^{13}$ C. Meny, P. Panissod, and R. Loloee, Phys. Rev. B 45, 12269 (1992).

${ }^{14}$ T. Fauster, G. Rangelov, J. Stober, and B. Eisenhut, Phys. Rev. B 48, 11361 (1993)

${ }^{15}$ J. Langford, Natl. Stand. Ref. Data Ser. (U.S., Natl. Bur. Stand.) 567 (1980).

${ }^{16}$ B. Warren, X-Ray Diffraction (Courier Dover, Dover, 1990).

${ }^{17}$ M. Krieger, "Herstellung Magnetischer Multilagen Mit Gepulster Laserablation-Charakterisierung Durch Elektrischen Transport," Ph.D. thesis, Universität Ulm, 2004.

${ }^{18}$ H. Errahmani, A. Berrada, G. Schmerber, and A. Dinia, Vacuum 56, 221 (2000).

${ }^{19}$ T. J. Jackson, S. B. Palmer, H. J. Blythe, and A. S. Halim, J. Magn. Magn. Mater. 159, 269 (1996).

${ }^{20} \mathrm{D}$. Attwood, Soft X-Rays and Extreme Ultraviolet Radiation (Cambridge University Press, Cambridge, 1999).

${ }^{21}$ D. C. Meyer and P. Paufler, J. Alloys Compd. 298, 42 (2000).

${ }^{22}$ J. Mathon, Lecture Notes in Physics (Springer, New York, 2001), p. 569. 